\title{
Middle lobe syndrome: An exceptional presentation of concomitant lepidic adenocarcinoma and bronchial anthracofibrosis
}

\author{
Shekhar Kunal1, Sudhir Jain², Ashok Shah'1 \\ 1Department of Pulmonary Medicine, Vallabhbhai Patel Chest Institute, University of Delhi \\ 2Department of Pathology, Nayati Medicity, Mathura, Uttar Pradesh, India
}

\begin{abstract}
Lepidic adenocarcinoma previously known as bronchioloalveolar carcinoma (BAC) is a non-small cell lung cancer with an indolent presentation. Bronchial anthracofibrosis (BAF) is caused by long-standing exposure to biomass fuel smoke often in poorly ventilated kitchen. Middle lobe syndrome (MLS) due to BAF is not uncommon however, lepidic adenocarcinoma then known as BAC, presenting as MLS has been documented only once before in the Polish literature. A 68-year-old never-smoker female with biomass fuel smoke exposure presented with cough and breathlessness. Imaging revealed MLS. Fiberoptic bronchoscopy visualised bluish-black hyperpigmentation with narrowing and distortion of right middle lobe bronchus suggestive of BAF. Transbronchial biopsy confirmed presence of lepidic adenocarcinoma. To our knowledge, this is the first detailed description of lepidic adenocarcinoma and BAF presenting as MLS.
\end{abstract}

Corresponding author: Prof. Ashok Shah, Department of Pulmonary Medicine, University of Delhi, Vallabhbhai Patel Chest Institute, Home address: F-168/ E, Rajouri Garden, New Delhi 110 027, India.

Tel. +91.11 .25433783 - Mobile +91.98101 .19117 .

E-mail: ashokshah99@yahoo.com

Key words: Biomass fuel smoke exposure; bronchial anthracofibrosis: lepidic adenocarcinoma; fibreoptic bronchoscopy; high resolution computed tomography.

Contributions: SK, AS, collected the clinical data, reviewed the literature; SJ, reviewed the pathological aspects; AS, worked on the concept and is responsible for the genuineness of the data. All the authors have drafted the manuscript and read and approved the final version.

Conflict of interest: The authors declare no conflict of interest.

Received for publication: 5 June 2017

Accepted for publication: 5 November 2017

CC Copyright S. Kunal et al., 2017

Tipografia PI-ME Editrice, Italy

Monaldi Archives for Chest Disease 2017; 87:864

doi: 10.4081/monaldi.2017.864

This article is distributed under the terms of the Creative Commons Attribution Noncommercial License (by-nc 4.0) which permits any noncommercial use, distribution, and reproduction in any medium, provided the original author(s) and source are credited.

\section{Introduction}

Lepidic adenocarcinoma is a form of non-small cell lung carcinoma and was formerly known as bronchioloalveolar carcinoma (BAC) [1], a term coined by Leibow [2] in 1960. There is a characteristic intra-alveolar pattern of spread and lepidic growth with an increased tendency for mucin production. It often has an indolent presentation with a peripheral location and is more commonly seen in middle-aged females and never smokers [3]. Radiologically, it has a diverse presentation and can range from focal to a diffuse form of the disease [4].

Cohen [5] in 1951 first described the presence of anthracotic pigmentation and narrowing of the airways. However, the term "BAF" was coined by Chung et al. [6] in their seminal description of 28 never smokers with significant wood smoke exposure. This clinical entity was predominantly seen in females [20/28] with tuberculosis being detected in 17. The authors postulated that tuberculosis was the causative agent and even advocated empirical initiation of antituberculous therapy in patients with BAF. However, evidence has accumulated to suggest that long-standing exposure to biomass fuel smoke is the most important risk factor in occurrence of BAF and that tuberculosis was a mere associated condition rather than the offending agent [7]. In developing countries such as India, BAF is predominantly seen among never smoker females with significant biomass fuel smoke exposure while cooking in poorly ventilated kitchens.

Middle lobe syndrome (MLS), a term coined by Graham et al. [8] in 1948, refers to chronic or recurrent collapse of the right middle lobe. However, it was Brock [9] who had first reported repeated episodes of right middle lobe collapse due to tuberculous adenopathy in 1937 and till date this is known as "Brock's syndrome". Though the MLS has an unmistakable radiological presentation, it can easily be missed on a postero-anterior chest radiograph. The specific stand-alone radiological presentation can be caused by a host of respiratory conditions and has continued to bemuse the clinicians.

We document a 68-year-old female with lepidic adenocarcinoma and BAF who presented to us with a MLS. Although the radiological finding of MLS is not uncommon in patients with BAF, till date there is only a single report of lepidic adenocarcinoma then known as BAC presenting as MLS in Polish language with a brief abstract in English [10]. To our knowledge, lepidic adenocarcinoma associated with BAF manifesting as MLS is yet to be documented.

\section{Case Report}

A 68-year-old HIV negative female was referred to our Institute for evaluation of complaints of cough and breathlessness for the past twoyears. Her clinical course was characterised by exertional dyspnoea 
and wheezing. Three months prior to presentation, she developed lowgrade fever, weight loss and right sided chest pain. In spite of several sputum stains and cultures for Mycobacterium tuberculosis being negative, the treating physician initiated the patient on standard first line anti-tuberculous therapy. Mantoux test too did not show any induration. Although the patient received eight weeks of anti-tuberculous therapy, she did not experience any relief, which prompted the referral. She was a never smoker but had cooked on biomass fuel in a poorly ventilated kitchen for the last four decades. She was exposed to biomass fuel smoke for 4-6 hours/day.

On examination, the patient was in no acute respiratory distress. There was no cyanosis, clubbing or pallor. Diaphragmatic excursion was comparable on both sides. Vesicular breath sounds of equal intensity with prolonged expiration and rhonchi were audible bilaterally. Oxygen saturation at room air was $98 \%$ and the total leucocyte count

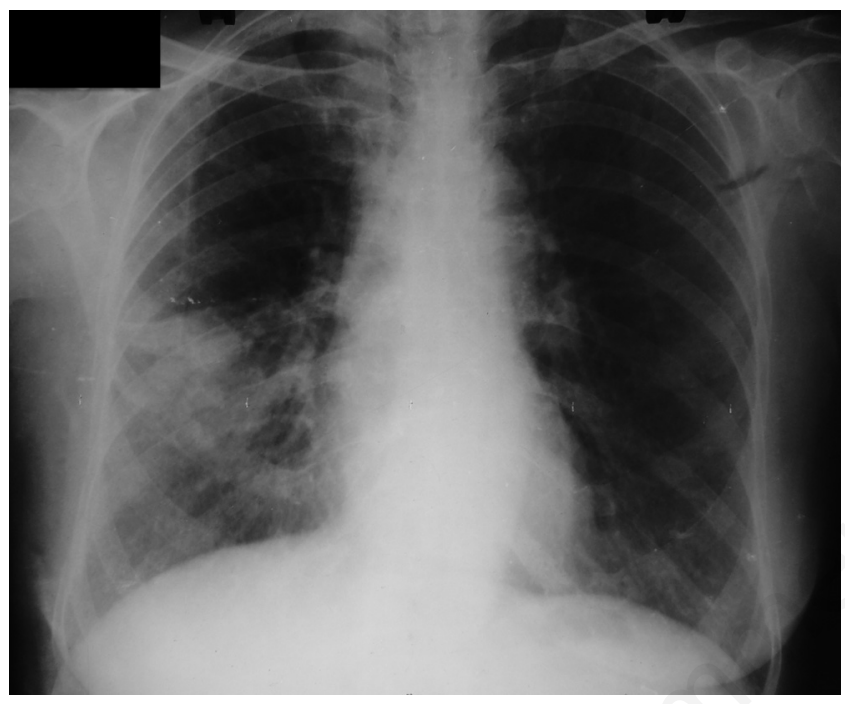

Figure 1. Chest X-ray postero-anterior view done 12 weeks prior to referral, showing an opacity in the right middle zone silhouetting the right cardiac border suggestive of a right middle lobe syndrome (MLS).

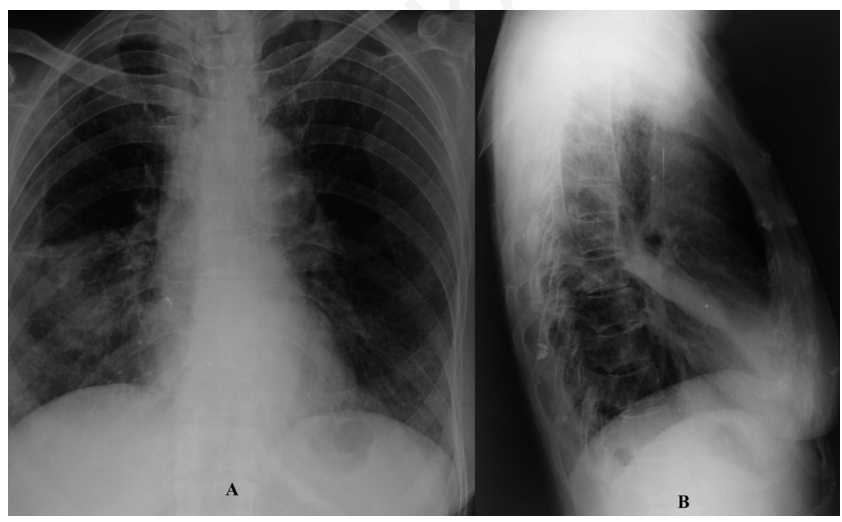

Figure 2. A) Chest X-ray postero-anterior view showing a poorly defined opacity in right mid and lower zones adjacent to the right cardiac border with loss of cardiac silhouette suggestive of MLS. B) Chest X-ray lateral view showing a sharply defined wedge shaped opacity extending into an antero-inferior direction from the hilum suggestive of MLS. was 5890 cells $/ \mathrm{mm}^{3}$ with neutrophils constituting $72 \%$. The ECG, echocardiogram, and blood chemistry were within normal limits. A review of the chest X-ray [postero-anterior (PA) view], done 12 weeks prior to referral, showed an opacity in the right middle zone silhouetting the right cardiac border (Figure 1) suggestive of a MLS. Chest radiograph (PA view) done on presentation too showed a similar picture (Figure 2A). The lateral view revealed a sharply defined wedge shaped opacity extending into an antero-inferior direction from the hilum pointing towards a diagnosis of MLS (Figure 2B). A contrast-enhanced high-resolution CT (HRCT) of the thorax showed a trapezoidal opacity with its base towards the hilum and approximating the right cardiac border (Figure 3: lung window] confirming the presence of MLS. Right paratracheal and sub-carinal lymphadenopathy were also seen. Sputum stains and cultures for Mycobacterium tuberculosis, fungi and other aerobic organisms were negative. There was no evidence of malignancy on sputum cytological examination. The patient was unable to perform spirometry but during the six-minute walk test, she walked 300 meters without desaturation. CT guided fine needle aspiration cytology from the middle lobe consolidation revealed monolayered sheets of round to oval cells with rounded nuclei, evenly dispersed chromatin and eosinophilic cytoplasm suggestive of malignancy (Figure 4A). Fibreoptic bronchoscopy (FOB) done to evaluate the MLS visualised dark bluish-black hyperpigmentation of the mucosa along with narrowing/distortion of the right middle lobe bronchus suggestive of BAF (Figure 4B). Stains and cultures of the bronchial aspirate were negative for $M$. tuberculosis and other aerobic organisms as well as pathogenic fungi. The aspirate was also negative for GeneXpert. Transbronchial biopsies from the right middle lobe revealed the presence of tumour cells arranged in alveolar and papillary structures, which confirmed the presence of lepidic adenocarcinoma (Figure 4C). A diagnosis of BAF with lepidic adenocarcinoma presenting as MLS was made. This was based on: i) characteristic imaging presentation suggestive of right MLS; ii) FOB finding of anthracotic pigmentation along with narrowing/distortion of right middle lobe bronchus; iii) FNAC and histopathological confirmation of lepidic adenocarcinoma. The patient was apprised of the treatment options. She was referred to a tertiary oncology centre for further management of lepidic adenocarcinoma. Subsequently, she was lost to follow-up and could not be contacted.

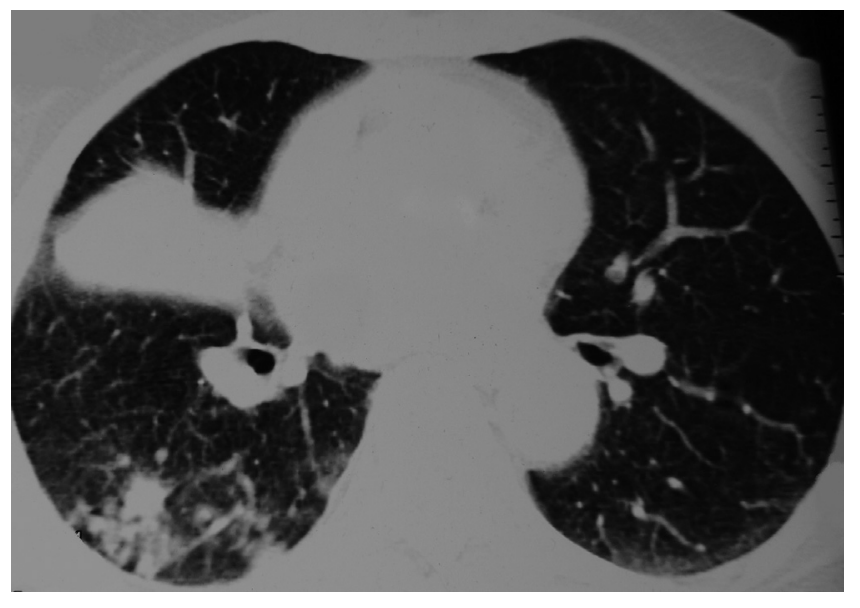

Figure 3. High resolution computed tomography of the thorax (lung window) showing a trapezoidal opacity with its base towards the hilum and approximating the right cardiac border confirming middle lobe syndrome. 


\section{Discussion}

Lepidic adenocarcinoma (previously known as BAC) (1) is an uncommon form of primary lung malignancy occurring in $4 \%$ of the NSCLC patients and is seen more frequently among middle-aged females, never smokers and people of Asian descent (3). The 2015 revised World Health Organization (WHO) classification of lung tumours (1) proposed for discontinuation of the term "BAC" and use of "lepidic adenocarcinoma" for this subgroup of lung malignancy. In the never smoker population, exposure to biomass fuel smoke is a risk factor for development of lung malignancies especially adenocarcinoma. Incomplete combustion of wood and coal leads to production of carcinogenic materials such as benzene 1,3 butadiene, formaldehyde and acetyldehyde (11). Parenchymal fibrosis and scarring are also well-established risk factors for lepidic adenocarcinoma (formerly BAC) [12]. Our patient developed BAF as a result of long-standing exposure to biomass fuel smoke leading to parenchymal fibrosis and scarring which could possibly have triggered the occurrence of lepidic adenocarcinoma.

Patients with lepidic adenocarcinoma could be asymptomatic or present with rather rare but characteristic symptom of bronchorrhoea, which is considered to be the hallmark of the disease [3]. The diverse but peculiar radiological presentation includes: i) solitary nodule or mass; ii) focal consolidation; and iii) multifocal or diffuse disease. The solitary pulmonary nodule is the most common CT imaging finding but foca//lobar consolidation is a well-recognised presentation. The two noticeable radiological signs associated with consolidative form of lepidic adenocarcinoma include: i) open bronchus sign [13]; and ii) CT angiogram sign which was once considered to be pathognomic for lepidic adenocarcinoma [4]. Unilateral crazy-paving pattern, an unusual HRCT feature, has been documented once [14].

BAF was first reported from India in a 65-year-old female with significant wood smoke exposure who presented with MLS [15]. FOB revealed anthracotic pigmentation and narrowing of the right middle lobe diagnostic of BAF. Bronchial aspirate cultured Mycobacterium tuberculosis and a diagnosis of BAF associated with tuberculosis presenting as MLS was made. The diagnosis of BAF is established when bluish-black anthracotic pigmentation along with narrowing and/or distortion of the affected bronchus is visualised on bronchoscopy. Multifocal bronchial narrowing when visible on HRCT of the thorax is a strong pointer towards the diagnosis of BAF. Current evidence suggests that tuberculosis is an associated condition rather than being the causative agent and has been reported in one-third of patients with BAF [7]. Apart from tuberculosis, BAF is now known to be associated with chronic obstructive pulmonary disease, lung malignancy and pneumonia [7,16].

MLS refers to the chronic/recurrent collapse of right middle lobe due to its anatomical predisposition. MLS is usually due to enlarged peribronchial lymph nodes, endobronchial mass or infective aetiology including bronchiectasis [17]. The other uncommon causes of MLS include BAF [15], allergic bronchopulmonary aspergillosis [18], endobronchial tuberculosis [19], pulmonary hydatid [20] and tracheobronchial amyloidosis [21]. Chest radiograph is the initial diagnostic modality for MLS and it presents as a homogeneous opacity abutting the right cardiac border with a loss of cardiac silhouette. The lateral view shows a characteristic wedge-shaped opacity extending anterior and inferior to the hilum [17]. On CT, MLS appears as a trapezoidal opacity with its base towards the hilum and contiguous with the right cardiac border, as was seen in our patient.

BAF most commonly affects the RML however, specific reason is yet to be elucidated. There is an increased deposition of carbon particles at the origin of RML due to angular take-off of the RML bronchus along with poor collateral ventilation and inadequate clearance of the carbon particles leading to impacted mucous. It is conceivable that all these factors could enhance the occurrence of pathological process, which leads to BAF in the middle lobe. A search of the literature on BAC/lepidic adenocarcinoma as a cause of MLS using the PubMed, IndMed and other databases found mention of a single report in the Polish literature. The authors described a 17-year-old female who presented with MLS and was diagnosed with BAC now known as lepidic adenocarcinoma [10]. However, detailed report is not available.

\section{Conclusions}

The 2015 revised WHO classification for lung cancer [1] proposed that the term BAC be discarded and replaced by lepidic adenocarcinoma. FOB done to evaluate MLS, in our patient, visualised BAF while TBLB revealed lepidic adenocarcinoma. Our patient had lepidic adenocarcinoma as well as BAF both of which are known to be associated with MLS. Since biomass fuel smoke exposure is a risk factor for occurrence of both BAF as well as adenocarcinoma, both these pathological processes may account for MLS in our patient. However, we were unable to ascertain whether MLS is due to lepidic adenocarcinoma or BAF or a combination of both. Our report highlights another exceptional explanation for the occurrence of MLS.

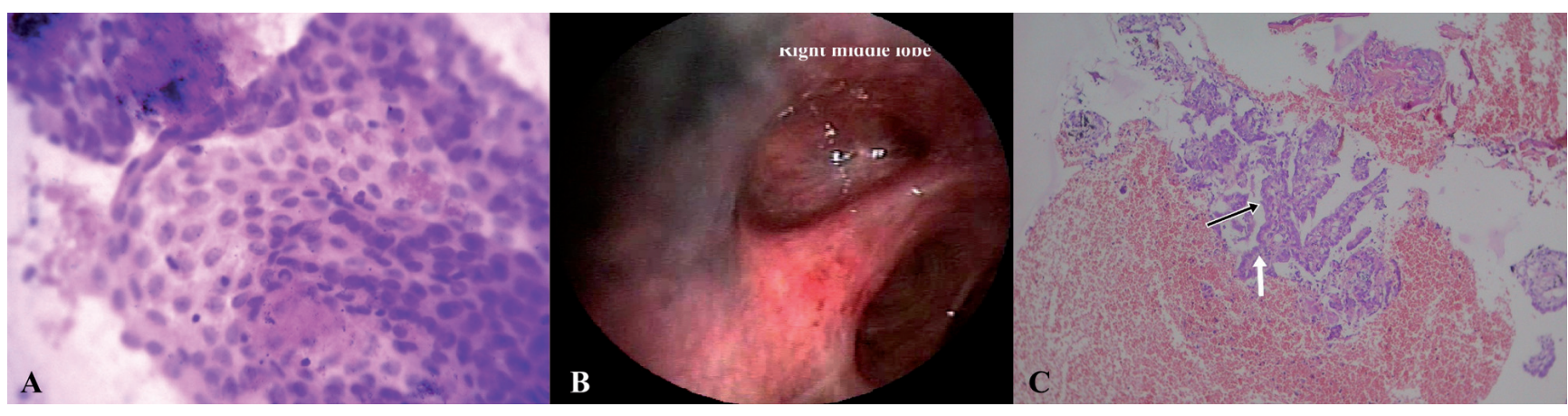

Figure 4. A) Low power view (X10) of the fine needle aspiration cytology from right middle lobe consolidation showing monolayered sheets of round to oval cells with rounded nuclei, evenly dispersed chromatin and eosinophilic cytoplasm suggestive of malignancy. B) Fibreoptic bronchoscopy (FOB) showing bluish-black hyperpigmentation of the mucosa along with narrowing/distortion of the right middle lobe bronchus suggestive of BAF. C) Low power view (X10) of the patient's biopsy specimen on H\&E stain showing tumour cells arranged in alveolar (black arrow) and papillary structures (white arrow) embedded in blood clots. 


\section{References}

1. Travis WD, Brambilla E, Nicholson AG, et al. The 2015 World Health Organization classification of lung tumors: Impact of genetic, clinical and radiologic advances since the 2004 classification. J Thorac Oncol 2015;10:1243-60.

2. Liebow AA. Bronchiolo-alveolar carcinoma. Adv Intern Med 1960;10: 329-358.

3. Raz DJ, He B, Rosell R, Jablons DM. Bronchioloalveolar carcinoma: a review. Clin Lung Cancer 2006;7:313-22.

4. Patsios D, Roberts HC, Paul NS, et al. Pictorial review of the many faces of bronchioloalveolar cell carcinoma. Br J Radiol 2007;80:1015-23.

5. Cohen AG. Atelectasis of the right middle lobe resulting from perforation of tuberculous lymph nodes into bronchi in adults. Ann Intern Med 1951;35:820-35.

6. Chung MP, Lee KS, Han J, et al. Bronchial stenosis due to anthracofibrosis. Chest 1998;113:344-50.

7. Gupta A, Shah A. Bronchial anthracofibrosis: an emerging pulmonary disease due to biomass fuel exposure. Int $\mathrm{J}$ Tuberc Lung Dis 2011;15:602-12.

8. Graham EA, Burford TH, Mayer JH. Middle lobe syndrome. Postgrad Med 1948;4:29-34.

9. Brock RC, Cann RJ, Dickinson JR. Tuberculous mediastinal lymphadenitis in childhood: secondary effects on the lungs. Guy Hosp Rep 1937;87:295.

10. Zalewska A, Kaznowska E, Kazirod T, et al. Bronchioloalveolar carcinoma in young people. Wspolczesna Onkologia 2002;6:391-4.

11. Barcenas CH, Delclos GL, El-Zein R, et al. Wood dust exposure and the association with lung cancer risk. Am J Ind Med 2005;47:349-57.
12. Aquino SL, Chiles C, Halford P. Distinction of consolidative bronchioloalveolar carcinoma from pneumonia: do CT criteria work? AJR Am J Roentgenol 1998;171:359-63.

13. Mir E, Sareen R, Kulshreshtha R, Shah A. Bronchioloalveolar cell carcinoma presenting as a "non-resolving consolidation" for two years. Pneumonol Alergol Pol 2015;83:208-11.

14. Pilaniya V, Kunal S, Jain S, Shah A. Image diagnosis: Bronchioloalveolar carcinoma presenting as unilateral "crazy-paving" pattern on high-resolution computed tomography. Perm J 2016;20:e111-2.

15. Kala J, Sahay S, Shah A. Bronchial anthracofibrosis and tuberculosis presenting as a middle lobe syndrome. Prim Care Respir J 2008;17:51-5.

16. Shah A. Bronchial anthracofibrosis: a perilous consequence of exposure to biomass fuel smoke. Indian J Chest Dis Allied Sci 2015;57:151-3.

17. Gudbjartsson T, Gudmundsson G. Middle lobe syndrome: a review of clinicopathological features, diagnosis and treatment. Respiration 2012;84:80-6.

18. Shah A, Gera K, Panjabi C. Childhood allergic bronchopulmonary aspergillosis presenting as a middle lobe syndrome. Asia Pac Allergy 2016;6:67-9.

19. Garg T, Gera K, Shah A. Middle lobe syndrome: an extraordinary presentation of endobronchial tuberculosis. Pneumonol Alergol Pol 2015;83:387-91.

20. Kunal S, Pilaniya V, Shah A. Middle lobe syndrome: a singularly rare presentation of complicated pulmonary hydatid disease. BMJ Case Rep 2016;pii:bcr2016214670.

21. Kunal S, Dhawan S, Kumar A, Shah A. Middle lobe syndrome: an intriguing presentation of tracheobronchial amyloidosis. BMJ Case Rep 2017;pii:bcr-2017-219480. 